\title{
¿INNOVAR? SÍ, PERO ¿CÓMO?
}

\author{
Goretti Cabaleiro \\ Profesora de Innovación y Estrategia, Universidad Alberto Hurtado
}

\begin{abstract}
Resumen
Con la proliferación de los Mercados de la Tecnología, las empresas ya no tienen la necesidad de desarrollar la tecnología de manera interna sino que también pueden adquirirla de manera externa. Sin embargo, lograr una combinación adecuada de I+D interna y externa es complejo. En efecto, los estudios científicos no entregan resultados concluyentes que permitan medir el impacto que tiene cada una de estas estrategias en el desempeño de las empresas. Este artículo describe las principales ventajas y desventajas asociadas a cada enfoque de acuerdo a la teoría y evidencia internacional.
\end{abstract}

\section{La creciente necesidad de innovar}

En los últimos años hemos sido testigos del rápido desarrollo de la tecnología, de la intensificación de la competencia, de cambios vertiginosos en las necesidades del cliente y de productos con un ciclo de vida cada vez más corto. Este panorama ha forzado a las empresas que quieren ser eficientes y competitivas a innovar constantemente y a mantenerse abiertas al cambio. Sin embargo, todavía no hay consenso sobre cómo hacerlo.

\section{I+D Interna}

Décadas atrás, una empresa innovaba principalmente basándose en los resultados de su departamento interno de investigación y desarrollo (I+D). Si los resultados eran prometedores, la empresa procedía a patentar o mantener en secreto este conocimiento que le permitía mantener una ventaja competitiva durante varios años. Sin embargo, hoy en día vemos cómo las empresas combinan diferentes métodos para innovar. Aparentemente, la innovación interna ya no es suficiente para ser competitivo.

\section{I+D Externa}

En los últimos años, la proliferación de los mercados de la tecnología ha abierto una variedad cada vez más amplia de oportunidades para que las empresas modernas innoven, lo que Ileva a que las estrategias de I+D sean cada vez más complejas y desafiantes (Arora, Fosfuri, y Gambardella, 2001). En la actualidad, las empresas no tienen por qué basar su capacidad innovadora en sus departamentos internos sino que también pueden "adquirir" tecnología o conocimientos de empresas externas.
En general, una de las opciones más utilizadas para la transferencia de tecnología son los contratos de licencia (Anand y Khanna, 2000b; Arora y Fosfuri, 2003). Estos contratos son acuerdos entre empresas a través de los cuales el propietario de la tecnología (licenciante) permite a otra empresa (licenciatario) producir, vender y utilizar una tecnología sin transferir su propiedad, a cambio de una compensación económica (Granstrand, 1999). En la última década, el volumen y la importancia de estos contratos ha aumentado notablemente. Por ejemplo, en los países del G8, el pago de royalties por transferencia de tecnología aumentó a una tasa anual promedio de 10,7\% desde 1980 hasta 2003 (Arora y Gambardella, 2010). Además, en varias industrias el manejo de estos contratos se ha convertido en una parte integral de la estrategia de las empresas para conseguir apropiarse de todo el valor posible de la innovación (Conti et al. 2013).

\section{¿Cómo asignar recursos entre la I+D interna y la I+D externa?}

Lograr el equilibrio entre la asignación de recursos para la innovación interna y la adquirida en el mercado de la tecnología es complicado por al menos dos razones. Primero, no hay consenso sobre cuál es la mejor estrategia entre las principales corrientes de la literatura, a saber, la literatura económica y la literatura de gestión estratégica. Segundo, no hay estudios concluyentes que analicen el impacto que tiene cada una de las estrategias en la economía de la empresa. En efecto, las consecuencias que las diferentes estrategias (patentar, licenciar o patentar y licenciar) tienen en el rendimiento de la empresa tanto a corto como a largo plazo no son concluyentes (Mulotte, Dussauge, y Mitchell, 2013; Wang, Roijakkers, y Vanhavrbeke, 2013). Sin embargo, indagar en 
éstos últimos puntos es fundamental para el diseño óptimo de las estrategias de I+D empresa nivel empresarial. Esto nos ayudaría a explicar cuando las empresas deben utilizar la opción de mercado, cuando deben confiar en las capacidades internas y como hacer mejores predicciones sobre el impacto de las estrategias de I + D en el rendimiento de la empresa (Walter, 2012).

\section{Mirada de la Economía}

La literatura económica hace hincapié en el largo plazo para fomentar una mejor división del trabajo y economías de aprendizaje. De acuerdo con esta lógica, las empresas deben especializarse en las actividades en las que gozan de ventaja comparativa y comercializar los productos de esas actividades (Arora \& Gambardella, 2010; Conti et al, 2013). Aunque esta literatura reconoce la transparencia limitada del mercado de la tecnología y sus ineficiencias (Arora et al., 2001), considera la tecnología como algo fácilmente replicable y trasladable (Lin, 2003). De esta manera, la economía promueve la separación entre la producción y el uso de la tecnología, también conocida como "división del trabajo del conocimiento" (Zhao y Chen, 2011). Esto es visto como una estrategia eficiente y socialmente óptima, porque cualquier otra solución podría aumentar potencialmente el costo total de la innovación y con ello afectar el bienestar de la sociedad en su conjunto. Por tanto, siguiendo el argumento económico, los acuerdos de licencia son complementarios y deseables debido a que estas empresas se benefician de aprovechar sus distintas competencias mientras acceden a los de otra empresa (Zhao y Chen, 2011). En general, el principio fundamental de esta literatura es que, a través de la especialización vertical, “El pastel global que las empresas producen en conjunto es mayor que si todas ellas hicieran investigación y comercialización» (Conti et al, 2013:. 753).

\section{Mirada de la Gestión Estratégica}

En oposición a la lógica económica, los defensores de la literatura de gestión estratégica avocan por un enfoque combinado de I+D. Siguiendo el enfoque de investigación abierta (Chesbrough, 2003), las empresas pueden y deben utilizar ideas externas y caminos externos en el mercado para complementar los esfuerzos de I + D de la empresa. La compra y la venta de la tecnología debe ser implementada de forma simultánea, como un medio para acelerar los procesos de innovación internos (Chesbrough y Bogers, 2014; Dahlander y Gann, 2010; Fosfuri y Tribó, 2008). A pesar de los importantes costos relacionados con la absorción de conocimientos, tiempo y atención gerencial (Knudsen y Mortensen, 2011; Laursen y Salter, 2006), se espera que la innovación abierta facilite el acceso a los recursos, los conocimientos y las competencias que de otro modo no estarían disponibles para la empresa (Faems, de Visser, Andries, \& van Looy, 2010; Gassman, Enkel, y Chesbrough, 2010; Huizingh, 2011). El supuesto subyacente en este caso es que la I+D propia y de abastecimiento externo son complementarios (Cassiman y Veugelers, 2006). En consecuencia, las decisiones sobre cómo innovar (I+D abierta vs I+D cerrada) deben ser tomadas de manera individual para así evaluar mejor los potenciales trade-offs de cada estrategia. Es decir, en lugar de perseguir una estrategia especializada en I+D, las empresas definirán la estrategia de innovación dependiendo de la naturaleza del problema para resolver (Pisano y Verganti, 2008).

\section{Evidencia: I+D Interna}

Cuando una empresa desarrolla la tecnología de manera interna le señala al mercado que es pionera en la industria en lo que se refiere a la creación de tecnologías que podrían resultar en productos y procesos innovadores. Las ventajas de ser pionero incluyen productos de mayor calidad, mayor diversidad y precios más bajos. Además, una empresa que demuestra destreza tecnológica a través de patentes le envía una señal al mercado acerca de sus "buenas prácticas de gestión". Esto demuestra no sólo la eficacia de sus inversiones tecnológicas (Zahra, 1996), sino también la capacidad de atraer y contratar a profesionales competentes y de fomentar un entorno innovador. Contrario a la situación de compra de licencias, la empresa tiene el control, la autonomía y la comprensión total sobre la tecnología patentada. Por tanto, es capaz de conservar la discrecionalidad en las decisiones estratégicas que son cruciales para su próximo desarrollo tecnológico y para conseguir una ventaja competitiva sostenida (Walter, 2012). De acuerdo con la teoría de la empresa basada en los recursos (Barney, 1991), la posesión de los recursos competitivos (patentes) y capacidades de innovación genera expectativas positivas sobre el desempeño de la empresa.

La I+D interna también genera menos incertidumbre en el mercado. Es decir,el uso de tecnologías desarrolladasinternamente es menos incierto que el uso de tecnologías adquiridas. En efecto, la I+D interna está contextualizada: no necesita ser traducida a las necesidades, las rutinas y las competencias de la empresa. El conocimiento está "situado" y definido en relación con un entorno físico y social determinado (Tyre \& Von Hippel, 1997). Por lo tanto, se espera que una tecnología desarrollada internamente pueda ser implementada y comercializada fácilmente. Ahora bien, puede ser que si escogemos la opción de desarrollar la tecnología de manera interna la empresa tarde más tiempo en lanzarla al mercado, perdiendo competitividad. 


\section{Evidencia: I+D Externa.}

La opción de licenciar podría reducir el tiempo de lanzamiento de un producto y permitir a la empresa lograr una mayor participación de mercado. Sin embargo, las fuentes externas de conocimiento implican varios costos, tales como los costos de búsqueda de la tecnología apropiada, de transacción, de entrada, de agencia y de integración (Arora, Fosfuri, y Roende, 2013; Arora y Gambardella, 2010) que pueden prolongar el desarrollo de nuevos productos o proyectos (Knudsen y Mortensen, 2011). Cuando se trabaja con tecnologías desarrolladas externamente, los licenciatarios deben adaptar e integrar estas tecnologías a sus productos específicos (Huston y Sakkab, 2006; Lin, 2003). Dado que los licenciatarios tienen acceso limitado a los conocimientos del licenciante (Mulotte et al., 2013), esto está lejos de ser una tarea sencilla, ya que depende de la capacidad del licenciador para transferir el conocimiento y la capacidad del licenciatario para absorber conocimiento externo (Ceccagnoli y Jiang, 2013).

\section{Conclusión}

Dada la importancia que tiene la innovación para el desarrollo económico, es clave entender cómo las empresas toman sus decisiones estratégicas, qué tienen en cuenta en el momento de hacerlo y cuáles son las consecuencias y costos asociados. Sin embargo, para hacer este análisis sólo se cuenta con información sobre la I+D que se encuentra en los reportes anuales, en noticias externas o en la cuenta de resultados. Como no es obligatorio para las empresas reportar sus contratos de licencia (ya sea que compren o vendan tecnología) y sólo salen a la luz los contratos que estratégicamente les interesan, estamos bastante lejos de llegar a una conclusión que nos indique cuál es la mejor estrategia y, en consecuencia, cómo innovar de manera eficiente. Por tanto, por el momento, las empresas deben considerar las alternativas disponibles y su buen juicio para determinar la mejor combinación de innovación interna y externa.

\section{Referencias}

Andries, \& van Looy, (2010). Technology Alliance Portfolios and Financial Performance: Value-Enhancing and Cost-Increasing Effects of Open Innovation. Journal of Product Innovation Management, 27(6):785-796.

Arora, A., Fosfuri, A., \& Gambardella, A. (2001). Markets for Technology: The Economics of Innovation and Corporate Strategy: MIT Press.

Arora, A., \& Gambardella, A. (2010). Ideas for rent: an overview of markets for technology. Industrial \& Corporate Change, 19(3), 775-803.
Anand, B., Khanna, T. (2000). Do Firms Learn to Create Value? The Case of Alliances. Strategic Management Journal, 21: 295-315.

Arora, A., Fosfuri, A. (2003). Licensing the Market for Technology. Journal of Economic Behavior and Organization 52: 277-295.

Arora, A., Fosfuri, A., \& Roende, T. (2013). Managing Licensing in a Market for Technology. Management Science, 59(5), 1092-1106

Barney, J. (1991). Special Theory Forum: The Resource-Based Model of the Firm: Origins, Implications, and Prospects. Journal of Management, 17(1), 97-99.

Cassiman, B., \& Veugelers, R. (2006). In Search of Complementarity in Innovation Strategy: Internal R\&D and External Knowledge Acquisition. Management Science, 52, 68-82.

Chesbrough, H. (2003). Open Innovation: The New Imperative for Creating and Profiting from Technology. Boston, Massachusetts: Harvard Business School Press.

Chesbrough, H., \& Bogers, M. (2014). Explicating open innovation: Clarifying an emerging paradigm for understanding innovation. In H. Chesbrough, W. Vanhaverbeke \& J. West (Eds.), New Frontiers in Open Innovation (pp. 3-28). Oxford: Oxford University Press.

Ceccagnoli, M., \& Jiang, L. (2013). The cost of integrating external technologies: supply and demand drivers of value creation in the markets for technology. Strategic Management Journal, 34, 404-425.

Conti, R., Gambardella, A., \& Novelli, E. (2013). Research on Markets for Inventions and Implications for R\&D Allocation Strategies. Academy of Management Annals, 7(1), 717-774.

Dahlander, L., \& Gann, D. M. (2010). How Open is Innovation? Research Policy, 39(6), 699-709.

Faems, D., de Visser, M., Andries, P., \& van Looy, B. (2010). Technology Alliance Portfolios and Financial Performance: Value-Enhancing and Cost-Increasing Effects of Open Innovation. Journal of Product Innovation Management, 27(6), 785-796. doi: 10.1111/j.1540-5885.2010.00752.x

Fosfuri, A., \& Tribó, J. A. (2008). Exploring the antecedents of potential absorptive capacity and its impact on innovative performance. Omega, 36(2), 173-187.

Gans and Stern (2003). The Product Market and the Market for "Ideas": Commercialization Strategies for Technology Entrepreneurs. Research Policy, 32(2), 333-350.

Gassman, O., Enkel, E., \& Chesbrough, H. (2010). The Future of Open Innovation. R \& D Management, 40, 213-221.

Granstrand, 1999). Granstrand, O. 1999. The economics and management of intellectual property:Towards intellectual capitalism: Edward Elgar Cheltenham, UK.

Huizingh, E. K. R. E. (2011). Open Innovation: State of The Art and Future Perspectives. Technovation, 31(1), 2-9. 
Huston, L., \& Sakkab, N. (2006). Connect and Develop: Inside Procter \& Gamble's New Model for Innovation. Harvard Business Review, 84(3), 58-66.

Knudsen, M. P., \& Mortensen, T. B. (2011). Some Immediate - but Negative - Effects of Openness on Product Development Performance. Technovation, 31, 54-64.

Laursen, K., \& Salter, A. (2006). Open for Innovation: The Role of Openness in Explaining Innovation Performance Among U.K. Manufacturing Firms. Strategic Management Journal, 27(2), 131-150.

Lin, B.-W. (2003). Technology transfer as technological learning: a source of competitive advantage for firms with limited R\&D resources. R \& D Management, 33(3), 327-341.

Mulotte, L., Dussauge, P., \& Mitchell, W. (2013). Does Pre-entry Licensing Undermine the Performance of Subsequent Independent Activities? Evidence from the global aerospace industry, 1944-2000. Strategic Management Journal, 34, 358-372.

Pisano, G. P., \& Verganti, R. (2008). What Kind of Collaboration Is Right for You? Harvard Business Review, 86(12), 78-87.
Tyre, M. J., \& Von Hippel, E. (1997). The Situated Nature of Adaptive Learning in Organizations. Organization Science, 8(1), 71-83.

Walter, J. (2012). The influence of firm and industry characteristics on returns from technology licensing deals: evidence from the US computer and pharmaceutical sectors. R \& D Management, 42(5), 435-454.

Wang, Y., Roijakkers, N., \& Vanhavrbeke, W. (2013). Learning-by-Licensing: How Chinese Firms Benefit from Licensing-In Technologies. IEEE Transactions on Engineering Management, 60(1), 46-58.

Zahra, S. A. (1996). Technology strategy and financial performance: Examining the moderating role of the firm's competitive environment. Journal of Business Venturing, 11(3), 189-219.

Zhao, G., \& Chen, W. (2011). Ensuring Quality Science from "R" to "D": An optimal Adoption Strategy for In-licensing of Pharmaceutical Innovation. IEEE Transactions on Engineering Management, 58(4), 643-661. 